\title{
EL PAPEL DEL PUERTO DE ALGECIRAS \\ EN LOS IMPACTOS RECIENTES DEL \\ CAMPO DE GIBRALTAR: PROTOTIPO \\ DE UN MODELO DE OCUPACION \\ DESARROLLISTA DEL ESPACIO LITORAL
}

\author{
Juan M. BARRAGAN MUÑOZ*
}

\section{OBJETIVOS}

En el presente trabajo se pretende calibrar la trascendencia que ha tenido el puerto de Algeciras en el proceso de transformación discrecional y voluntarista de una comarca económicamente deprimida como es la del Campo de Gibraltar. Es fácil de suponer que tales cambios se advierten en mayor medida en la franja litoral. Las características naturales de la parte interior, la orientación de la actividad industrial y la localización de los principales asentamientos, explican la reproducción de un modelo de desarrollo selectivo muy concreto que utiliza la franja litoral como soporte territorial.

La hipótesis de partida podría ser, en consecuencia, demostrar como el puerto de Algeciras ha sido el instrumento básico utilizado en una determinada política desarrollista que ha transformado radicalmente, y deteriorado gravemente, el espacio natural y cultural señalado. El marco temporal que encuadra los cambios acontecidos es de magnitudes reducidas, ya que en apenas dos décadas, 1965-1985, el Campo de Gibraltar es testigo de una serie de alteraciones económicas, medioambientales, sociales y culturales mayores que las conocidas en el resto de toda su historia.

La coyuntura que propicia un modelo de desarrollo muy concreto deriva de una serie de factores, a los que se hará referencia más adelante, que bien podrían agruparse por su naturaleza en: de Desarrollo Regional, Políticos, y Locacionales.

* Profesor Titular de Análisis Geográfico Regional. Universidad de Cádiz. 
Los resultados, identificados y agrupados en impactos directos y efectos indirectos, constituyen una inmejorable muestra del precio que los ciudadanos aquí residentes han tenido que pagar por un intento frustrado de incorporarse al tan deseado y necesitado desarrollo.

\section{EL CAMPO DE GIBRALTAR EN LA DECADA DE LOS AÑOS SESENTA: ENTRE LA POLITICA INTERNACIONAL Y EL DESARROLLO REGIONAL}

Sin intención de dramatizar y mucho menos reducir a un simple bosquejo lo que en realidad era una compleja y cruda vivencia para los habitantes de ésta comarca, la situación de los años sesenta se caracterizaba por una economía dependiente y en proceso de desestructuración, por problemas muy graves de infraestructura, una renta percápita de las más bajas de España, etc.

Buena prueba de ello lo constituía un sector primario latifundista, extensivo, arcaico y en no pocas ocasiones improductivo; el sector secundario era casi inexistente y reducido en todo caso a la transformación de productos relacionados con la pesca y la alimentación en general; el sector terciario se reducía a los servicios más básicos; la escasez de viviendas, agua, energía eléctrica, etc., así como la carencia de vías de comunicación completaban un panorama desolador, tan sólo comparable al ofrecido por las comarcas más deprimidas de Andalucía e incluso del resto del país.

La red urbana formada por éstos municipios organizaban, desde el punto de vista funcional, un cinturón de núcleos dependientes de las necesidades de mano de obra y servicios de uno de los mayores fracasos políticos de la dictadura franquista: Gibraltar. La ocupación británica suponía un difícil reto para el que no parecía vislumbrarse solución alguna. Es muy probable que se recurriera al desarrollo de la comarca utilizando en éste caso dicho concepto como instrumento prioritariamente político.

Por otra parte, en ésta época, primera parte del decenio, industria era sinónimo de desarrollo. Al mismo tiempo, en España, estaban en pleno apogeo las teorías de Perroux, sobre polarización y difusión industrial. También estaba muy extendido el modelo occidental de localización de industris básicas y pesadas.

La revolución del transporte marítimo, medios de propulsión primero, carrera hacia el gigantismo después, permitieron que los costes por unidad transportada descendieran de forma considerable, haciendo rentable el acarreo de ciertos productos hasta factorías con facilidades portuarias. El incremento de la demanda de petróleo y otras materias primas imprescindibles (fosfatos, hierro, carbón, etc.) es sólo la consecuencia del proceso descrito. 
Era, pues, logico suponer que en nuestro país también se iba a extender el modelo industrial portuario que en Europa Occidental había proliferado inmediatamente después de la última conflagración bélica. Las denominadas ZIP (Zones Industrialles Portuaires) en la terminología francesa, o MIDA (Maritime Industrial Development Area) en la anglosajona, se extendieron por España rápidamente: Tarragona, Huelva, Cartagena, etc. son buenos ejemplos. El puerto de Algeciras puso las bases para que dicho modelo se repitiera en el Campo de Gibraltar.

Con tales antecedentes se diseña uno de los proyectos mejores, teóricamente al menos, cuyo objetivo era mejorar el nivel de vida de los ciudadanos campogibraltareños. Nace el Plan Integral de Desarrollo del Campo de Gibraltar. Entre 1965 y 1975 se conceden ayudas para mejorar la agricultura, (Plan de Colonización del Guadarranque), ganadería, silvicultura, industria, vivienda, educación, etc. La declaración de Zona Preferente de Localización Industrial Agraria y Zona Preferente de Localización Industrial permitió encauzar inversiones públicas y privadas (no siempre de manera voluntaria como en el caso de CEPSA que prefería Bilbao antes que la Bahía de Algeciras) hacia la comarca.

\section{LOCALIZACION PORTUARIA DE LAS INDUSTRIAS BASICAS Y PESADAS COMO REFLEJO DE UN MODELO DE OCUPACION LITORAL}

Los hechos que estamos describiendo culminan con la orientación de casi todas las inversiones hacia un tipo muy determinado de industrias. En efecto, núcleos fabriles de enclave son los que van a dominar la génesis del desarrollo industrial: química básica, petroquímica, metalurgía, celulosa, etc.

$\mathrm{La}$ ausencia de materias primas que transformar y energía que sustente dicho proceso son las causas que explican tal comportamiento. A la vez se produce una puesta en valor de la situación intercontinental e interoceánica de la Bahía de Algeciras. También sus características naturales, ideales para el desarrollo portuario: calado, abrigo, amplitud de superficie marítima y terrestre disponible, son consideradas de forma excepcional.

La ubicación de las distintas factorías, en polígonos industriales situados casi siempre al borde del mismo mar, es buena prueba de que sus necesidades de materias primas y energía, así como la salida de productos acabados o semielaborados, son satisfechas a través de medios de transporte marítimo. Por tanto, el desarrollo portuario no se hace esperar, ya que es consecuencia de la coyuntura descrita. Acerinox, Cepsa, Crinavis, etc. serán algunas de las sociedades directamente responsables de los cambios producidos a iniciativa de la Administración Pública. 


\section{EL PUERTO DE ALGECIRAS-LA LINEA: TRAFICO Y ESPECIALIZACION FUNCIONAL}

El desarrollo industrial y económico que sucederá a ésta situación trae aparejado consigo unos cambios más o menos visibles en el territorio. Uno de los que mayor impacto visual genera es, precisamente, el producido por la ampliación de las instalaciones portuarias. El paisaje se va endureciendo sucesivamente con la aparición de superficies creadas por rellenos del mar.

Las instalaciones físicas del puerto se ampliaron, como se verá más detalladamente, de una forma considerabilísima. Lo que en 1940 era poco más que un espigón, el rompeolas de Isla Verde, y una pequeña terminal de atraque, donde ahora aparece el muelle de pasajeros de la Galera, es en la actualidad una compleja instalación portuaria que se reparte por todos los términos municipales costeros de la comarca. Por añadidura, es posible afirmar que el futuro no puede ser más halagüeño. Es, y será, por excelencia uno de los mayores y más importantes puertos de España y, por supuesto, de la región.

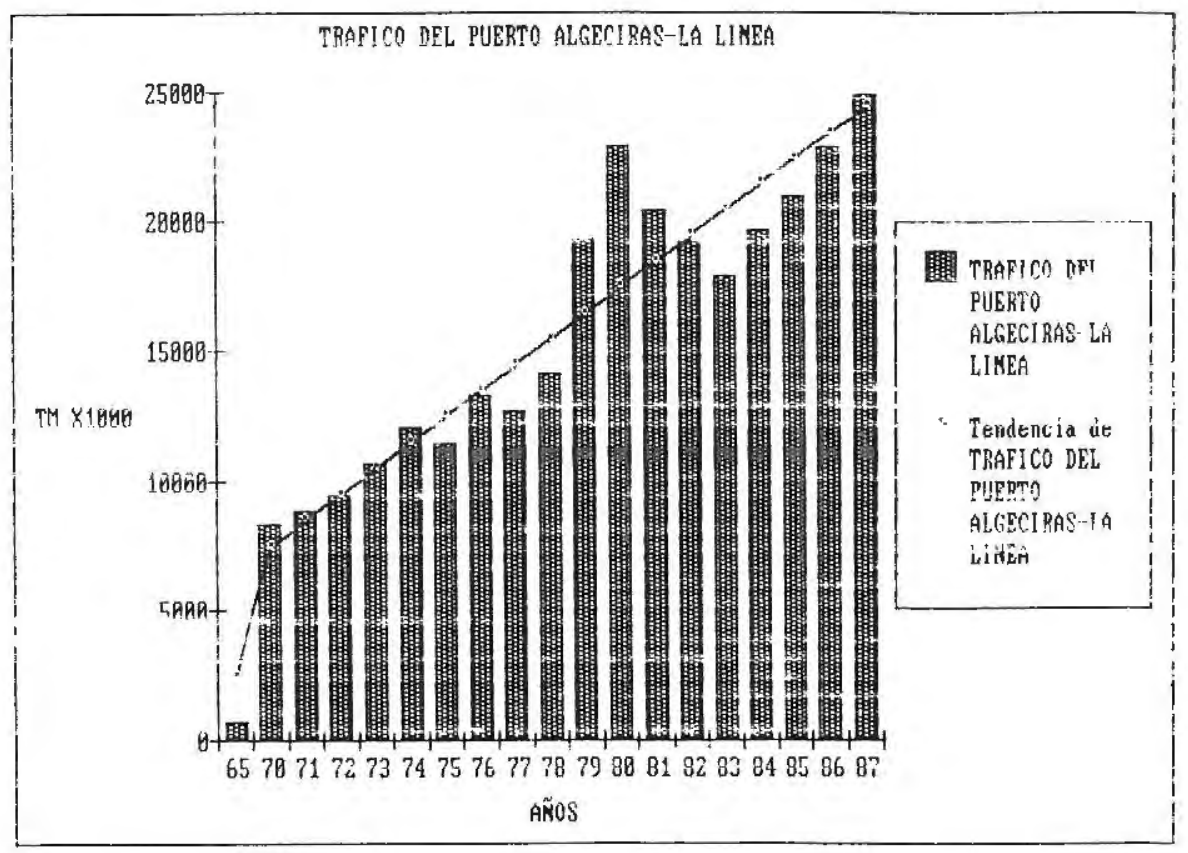

FIFURA A- EVDLUL ION Y TENDENCIA DEL TRAFICO DRET:IART 
Su carácter polinúclear y multifuncional han permitido el lógico incremento de tráfico. Este debe ser tildado, como mínimo, de espectacular. En 1965 apenas conseguía alcanzar el medio millón de toneladas. El ritmo de negocio marítimo aumenta de forma súbita amparado en el vertiginoso proceso industrial: en 1985 se lograba multiplicar la cifra inicial por más de 40. En los últimos ejercicios no resulta extraño que se superen los 25 millones de toneladas.

La especialización funcional se resuelve de la siguiente manera: casi el 70 $\%$ del tonelaje son graneles líquidos, alrededor del 6-7 \% graneles sólidos y el resto, en torno al 23-24\% mercancía general, en su mayor parte, contenedorizada.

Algeciras destaca en el sistema portuario nacional y regional en las siguientes funciones: petróleos y derivados, contenedores en régimen de transbordo internacional, gracias a su excelente localización en las rutas interoceánicas, el binomio pasajeros-automóviles que cruzan el Estrecho por éste «puente» euroafricano, y en menor medida pesca.

\section{INCIDENCIA EN EL ESPACIO LITORAL DEL PUERTO Y SUS FUNCIONES}

No es fácil establecer metodológicamente un esquema que aglutine, de forma lógica, los impactos y efectos que un puerto genera en el territorio. Se trata, en definitiva, de un ejercicio taxonómico, clasificatorio, pero que puede ayudar a comprender cual es la trascendencia de un puerto, y sus funciones, en el proceso de ocupación, utilización y estructuración del espacio litoral.

El esquema propuesto, original en su planteamiento para las infraestructuras de transporte, y diseñado especialmente para los sistemas portuarios, se ha ensayado anteriormente de una forma sintética para el subsistema portuario andaluz. Dicho esquema parte de la diferenciación que puede hacerse entre las consecuencias derivadas de la propia existencia de una infraestructura física de transporte como es el puerto, a las que agruparemos bajo la expresión de impactos directos, de aquéllas originadas por cualquiera de las funciones portuarias, a las que denominaremos efectos indirectos.

En definitiva, de lo que se trata es de establecer las pertinentes diferencias entre la incidencia del puerto y de sus respectivas funciones en el territorio. La utilización del método utilizado puede ser muy útil en las fases de análisis y diagnóstico de cualquier plan de ordenación territorial. 


\subsection{Impactos directos}

Como es lógico suponer los impactos directos pueden agruparse a su vez en los distintos subsistemas territoriales clásicos: a) físico-natural, b) económico-productivo, y c) urbano-relacional. Con dicho enfoque el análisis territorial del puerto de Algeciras, sin ánimo de ser exhaustivos, no resulta difícil.

a) Dentro del subsistema físico-natural se observan una serie de impactos producidos claramente por el puerto entre los que cuenta, en primer lugar, el más básico: el hecho de que la administración portuaria pase a ser propietaria de todo el perímetro costero de la Bahía homónima; desde Punta Carnero, en un extremo del arco ribereño, hasta casi la misma frontera con Gibraltar, en el otro. Hacemos referencia a la propia detentación de la propiedad física, con todo lo que ello implica en relación con las futuras posibilidades de alteración del medio natural para determinados usos.

Otro de los aspectos más relevantes que aparece es el impacto producido en el medio natural por la construcción del puerto. El modelo más repetido de obra de ingeniería portuaria en España ha sido conseguir las superficies necesarias a costa de ganarle terreno al mar. El endurecimiento y degradación del paisaje, la alteración de las corrientes marinas por diques o espigones, etc. son algunos de los impactos más directos que se pueden enumerar.

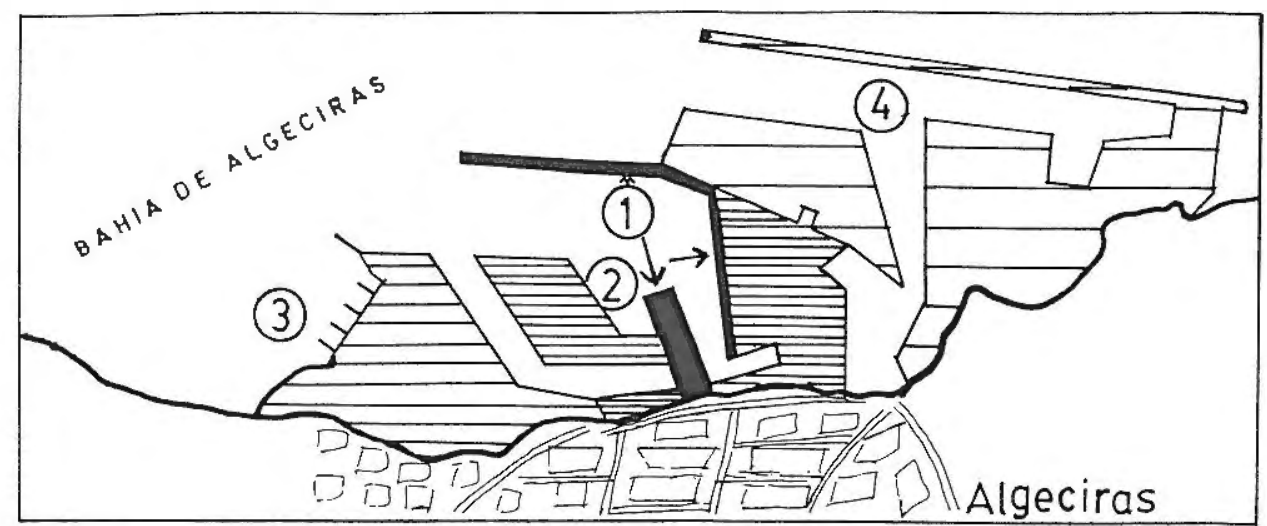

FIGURA B- EVOLUCION DE LA SUPERFICIE RELLENADA EN EL PUEFTO DE ALGECIRAS: 1 - en traina negra HASTA 1940, 2- EL FUERTO EN LA ACTUALIDAD (rallado $f i n a)$, J-RELLENOS PREVISTOS PARA EL MUELLE DE SANTIAGO, 4- FUTURO FUERTO EXTERIOR

El puerto de Algeciras es el principal agente generador de espacio físico. La observación del plano de las instalaciones no permite dudas. Casi toda la superficie terrestre ha sido creada artificialmente. En apenas quince años se han rellenado $140 \mathrm{Ha}$. aproximadamente: en 1973 la superficie terrestre del 
puerto era de apenas $50 \mathrm{Ha}$, en 1988 alcanza $190 \mathrm{Ha}$. repartidas de la siguiente forma: relacionada con muelles del servicio 97, con muelles de utilización particular 78 (incluyendo las $45 \mathrm{Ha}$. de relleno de CRINAVIS, que nunca tuvieron utilidad alguna), a las que habría que añadir otras casi $15 \mathrm{Ha}$. entre accesos, diques, etc.

Si ésta es la descripción de lo ocurrido en la historia más reciente, el panorama debe completarse con los últimos rellenos concluidos en 1987: 14,5 Ha. en el Muelle del Navío y 7,7 Ha. de la Dársena Pesquera. El impacto producido por los rellenos continuará habida cuenta de los proyectos a medio y largo plazo: $55 \mathrm{Ha}$. para estacionamientos en el denominado Muelle de Santiago y entre 100-150 Ha. para la ampliación del Puerto Exterior respectivamente.

b) En el Subsistema económico-productivo el impacto de la construcción y funcionamiento de un puerto es mucho menor, como veremos más adelante, que si consideramos las consecuencias de las propias funciones portuarias. De todas formas, y prescindiendo de la mano de obra ocupada durante la fase de construcción, 100-200 trabajadores, entre uno y tres años dependiendo de la envergadura de la obra, la masa laboral ocupada más fácilmente cuantificable es la plantilla fija del propio puerto: unas 200 personas.

c) El Subsistema urbano-relacional se ve afectado de una forma directa y contundente ya que, en primer lugar, el puerto posee una estructura polinuclear, repartiendo su incidencia por todos y cada uno de los núcleos urbanos costeros de la comarca, en unos con mayor incidencia que otros. En segundo lugar, el puerto forma parte de las ciudades, claramente en los casos de Algeciras y Tarifa; es decir, son puertos urbanos o ciudades portuarias, según se mire. Los impactos derivados en éstas dos ciudades son:

1) Efecto barrera, al dificultar o impedir al ciudadano el acceso directo, el disfrute, al mar. La infraestructura portuaria constituye una barrera, supuestamente, infranqueable para la población ajena a las actividades portuarias como señala el Decreto de 11-XII-1942. Las Avenidas Virgen del Carmen en Algeciras y Alcalde Juan Núñez en Tarifa constituyen realmente el límite físico entre el mar y la ciudad, al encontrarse entre ambos el recinto portuario protegido por la correspondiente verja o pared. Estas últimas son de vital importancia pues condicionan la dirección de los ejes circulatorios de esta parte de la ciudad. Del mismo modo, la línea férrea que enlaza con el puerto de Algeciras hace las veces de barrera separadora claramente visible. También para la expansión urbana el puerto constituye una barrera, aunque hay que reconocer que la ciudad encorseta el crecimiento del propio puerto hacia tierra, lo que le obliga a entrar en la dinámica constructiva del relleno. 
2) El efecto pantalla se produce cuando entre el mar y las posibilidades de observación aparezca una infraestructura portuaria que no sólo degrade o endurezca el paisaje sino que incluso impida verlo. Es el caso de Algeciras y Tarifa cuando los diques, terminal de contenedores o estación de lanchas rápidas impiden ver el paisaje marítimo.

\subsection{Efectos indirectos}

Los efectos indirectos son los causados por las propias funciones portuarias: comercial, industrial, pesquera, etc. Cronológicamente son posteriores al hecho de la construcción de la infraestructura portuaria propiamente dicha. Igual que con los impactos directos se pueden agrupar en los distintos subsistemas que configuran el territorio. La importancia de éstas suele ser mayor incluso que las generadas por la misma infraestructura.

a) Dentro del Subsistema físico-natural los efectos básicos de la existencia de una serie de funciones portuarias, donde destaca la industrial, son sentidos con toda crudeza en el Campo de Gibraltar. Polución del aire, contaminación de las aguas, ruidos, etc. son algunos de los efectos más generalizados en la zona, y que son provocados por centros como los de Cepsa, Celupal, Lubrisur, Interquisa, Petresa, Acerinox, Gibraltar Intercar, etc. No es posible olvidar que buena parte de las inversiones del Plan Integral de Desarrollo estuvieron destinados a la industria química y petroquímica.

En la actualidad el fenómeno de la degradación del medio ambiente, debido a los residuos de determinados procesos industriales, es posible seguirlo a través de los medios de comunicación. La intensa actividad de los grupos ecologistas en los dos últimos años se encarga de ello. Así no resulta extraño encontrar titulares periodísticos de la prensa local tales como:

«Por presunto delito ecológico en la Bahía de Algeciras, el Ayuntamiento de San Roque, pide prisión menor para el director de CEPSA», «Ochenta vecinos visitaron la refinería "Gibraltar" de CEPSA», «En protesta por la contaminación de la Bahía de Algeciras, miembros de "Greenpeace" realizan una protesta en el pantalán de la refinería de CEPSA», «Una expedición marina, desde San Roque hasta Algeciras, se manifestó contra el deterioro medioambiental», «En la térmica de Puente Mayorga, AGADEN despliega una pancarta de protesta en una chimenea industrial», etc.

A estos titulares deben añadirse otros al final del verano de 1989 que hacen referencia a los problemas generados por el olor despedido por algunas fábricas de harina de pescado que han venido a completar el panorama descrito por la industria química o petroquímica. 
Puede afirmarse sin temor a error que la mayor parte de la contaminación del litoral está relacionada con las industrias portuarias. Las molestias que cotidianamente soporta la población junto a la misma concienciación que los grupos ecologistas producen, con sus cada vez más numerosas, espectaculares e incluso arriesgadas acciones, está generando en el Campo de Gibraltar un estado de opinión pública sólo repetido en casos tan paradigmáticos, desde el punto de vista medioambiental, como Huelva o la Ría de Bilbao.

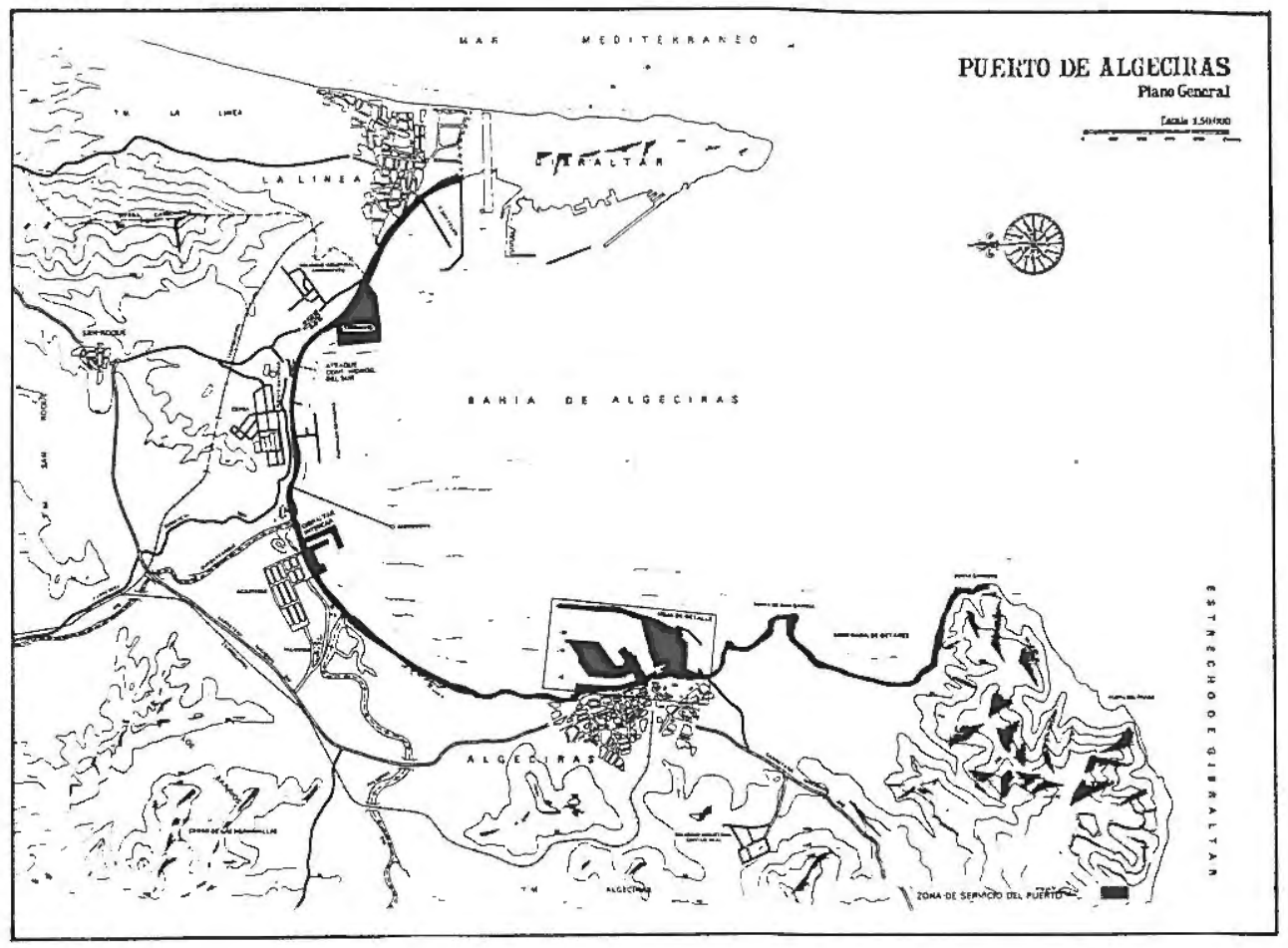

FIGURA C- INSTALACIONES FORTLIARIAS DE LA BAHIA DE AL SECIFAS

La degradación del medio ambiente se manifiesta de muy diversas formas: desaparece la pesca en la Bahía de Algeciras, se incrementan las dermatitis producidas por los baños en las playas de la zona, arrecian los procesos asmáticos, etc. Durante el verano de 1989 ha sido frecuente la catalogación negativa de algunas playas, llegando incluso a desaconsejar el baño por falta de calidad del agua y de la arena.

Desde hace más de dos décadas la actitud de la Administración, Central primero y Autonómica después, ha sido permisiva y connivente. Afortunadamente, desde hace sólo algunos meses la Agencia del Medio Ambiente de la 
Junta de Andalucía ha adoptado una actitud relativamente drástica ante la trascendencia del problema, advirtiendo de un posible cierre en caso de no redactar y cumplir el tan necesitado Plan Corrector de Vertidos.

Hay que reconocer aquí un cambio importante y beneficioso por parte de la Administración medioambiental provincial de la Comunidad Autónoma, que, sin embargo, resulta obvio, por la gravedad del asunto, está necesitada de la continuación de apoyo político desde las más altas instancias.

b) En el Subsistema Económico-Productivo es donde se concentran los efectos más beneficiosos de las funciones portuarias industriales, aunque también, si llevamos a cabo un análisis más riguroso, las deficiencias estructurales más preocupantes.

Es necesario señalar como casi todas las grandes empresas son portuarias y dependen del transporte marítimo para importar las materias primas, exportar los productos manufacturados o ambas cosas a la vez: Cepsa, Acerinox, Celupal, etc. En un estudio encargado a ICSA se concluía que en torno a 35.000 personas, casi el $50 \%$ de la población activa de la comarca, dependían directa $o$ indirectamente de las funciones portuarias.

La estructura del empleo industrial está casi modelada por la existencia del puerto y sus respectivas funciones, ya que a ellas se debe más del $90 \%$ del total. Si se clasifican los pucstos de trabajo en tres grandes grupos industriales: de importación de materias primas, de exportación de productos elaborados y de enclave, se observa como éstas últimas, con casi el $55 \%$, predominan sobre el resto, sobre todo si se tiene en cuenta que el segundo grupo cuenta con cerca del $30 \%$ de los efectivos dedicados a procesos relacionados con la alimentación humana y pesca.

c) Subsistema urbano-relacional; si de una forma breve tuviéramos que resumir la situación respecto a este apartado podría afirmarse sin temor a error que las funciones portuarias son las actividades que causan los mayores problemas a la ciudad, de Algeciras especialmente. Congestión de accesos y saturación de vías y ejes intraurbanos constituyen dos de las principales preocupaciones del Ayuntamiento en relación con la existencia de un puerto urbano.

A todo el flujo de emigrantes norteafricanos, verdadera avalancha estival, los turismos españoles o del resto de países de la CEE con destino a Ceuta o Marruecos, como tráfico ligero, se le añade el tráfico pesado cuyo origen/ destino está precisamente en el puerto de Algeciras o en cualquiera de las instalaciones industriales del arco ribereño. En éste último sentido es posible constatar que más del $40 \%$ del tráfico pesado está relacionado con las funciones portuarias. Precisamente el origen de obras como la Ronda intermedia de Algeciras que enlaza con la N-340, mejora de los accesos, etc. se orienta a solucionar uno de los problemas más graves de la ciudad. 
Por último en no pocas ocasiones la infraestructura industrial-portuaria es la responsable de cambios drásticos en la organización del sistema urbano existente, sobre todo en la base de la red jerárquica.

Así es posible mencionar como ejemplo el hecho de que la pequeña aldea de Palmones, núcleo de pescadores de la Bahía, quedó asfixiada, junto al Centro de Interés Turístico Nacional de Guadacorte, por la planta industrial de Acerinox (67 Ha.). Según LOZANO (1983, pp. 371) «el tema de la aldea de Palmones levantó una ola de protestas solamente acalladas por el autoritarismo de la época». Del mismo modo, el siempre inactivo astillero de CRINAVIS $(60 \mathrm{Ha}$.) tomó para su relleno unos terrenos que habían estado ocupados tradicionalmente por urbanizaciones y casas semidispersas.

\section{CONCLUSIONES}

El Campo de Gibraltar constituye uno de los mejores ejemplos de ternitorio marcado profundamente por la influencia de las infraestructuras de transporte, destacando las marítimas de forma especial. En éste caso, el puerto se ha encargado de estructurar no sólo la economía de la sociedad campogibraltareña, también la organización del territorio, red urbana e incluso espacios intraurbanos han sido condicionados en buena medida por la existencia del puerto y sus funciones.

Es probable que la metodología aplicada sea útil en otros casos. Estamos seguros de que el planteamiento, tal y como ha sido expuesto, sirve para estudiar, tan detalladamente como sea necesario, de forma lógica y ordenada, la incidencia de un puerto en el área donde ejerce un cierto grado de influencia.

La distinción entre los impactos derivados de la existencia del puerto, tomado éste como ente físico, y las consecuencias de sus funciones, contribuye a precisar distintos procesos causa-efecto en las relaciones puerto-territorio. En el ejemplo que se ha tomado como referencia no ha sido posible profundizar con el detalle deseado. No obstante, parece evidente que las líneas maestras del ensayo metodológico han sido diseñadas de forma que se ha puesto de manifiesto la importancia del puerto en la organización del territorio en una comarca andaluza donde éste ha sido un elemento transformador de primera magnitud.

Para la deseada integración entre la construcción de las infraestructuras portuarias y las peculiaridades de cada territorio resulta imprescindible conocer las posibilidades de relación mutua entre éstos dos sistemas interactivos. Sólo así la planificación territorial podrá localizar y prevenir efectos no deseados. 


\section{BIBLIOGRAFIA}

ALONSO FRANCO, F.: «EI transporte marítimo en Andalucía», Transporte y Desarrollo Regional en Andalucía, Instituto de Desarrollo Regional, Universidad de Sevilla, n. ${ }^{\circ} 17,1979$, pág. 183206.

ALVARGONZALEZ, R.: «Funciones y morfología en los puertos españoles», Rev. Ería, n. ${ }^{\circ} 8,1985$, pág. 5-59.

BARRAGAN MUÑOZ, J.M.: «Incidencia territorial de la política portuaria en Andalucía», Geografía de Andalucía, Dir. Cano, G., vol. VII, 1989, (en prensa).

BARRAGAN MUN̈OZ, J.M.: «La infraestructura portuaria y la OT: una nueva etapa en la Ordenación del Territorio sectorial», Revista de Estudios Territoriales, n. ${ }^{\circ} 28,1989$, pág. 111-119.

BECHTEL CIVIL INC.: Bahía de Algeciras. Development Program, 1986, 141 pp.

BULK SYSTEMS INTERNATIONAL: «Enviomment comes first at Los Barrios», Bulk Systems International, vol. 15, n. $^{\circ} 12,1984$, pág. 49-51.

CENTRO DE ESTUDIOS Y EXPERIMENTACION DE OBRAS PUBLICAS (CEDEX): Evaluación metodológica del impacto ambiental de un puerto deportivo, Madrid, MOPU, 1987, 42 pp.

CENTRO DE ESTUDIOS TERRITORIALES Y URBANOS DE LA JUNTA DE ANDALUCIA: Estudio del Area de Influencia del Puerto de Algeciras, Sevilla, Consejería de Obras Públicas y Transportes, 1987, 245 pp. (fotoreproducción).

CINCO DIAS: «Sea Land se queda en Algeciras», Diario Cinco Dias, 31 de julio de 1984, pág. 24.

COLONELLO, R.: «El impacto de los nuevos servicios de líneas regulares alrededor del mundo», Marina mercante y transporte marítimo, UIMP, Santander, 1985, 4 pp.

CORTES MENDOZA, J.M.: El puerto de Algeciras: funcionalidad en el Mediterráneo e influencia local y comarcal, Málaga, Facultad de Ciencias Económicas, 1988, 87 pp. (fotoreproducción).

DIRECCION GENERAL DE PUERTOS Y COSTAS (DGPC): Metodología para la evaluación de proyectos de inversión en puertos, Madrid, MOPU, 1981, 78 pp.

DGPC: Plan General de Actuación 1982, Madrid, MOPU, 1982.

DGPC: Plan General de Puertos 1985-1990, Madrid, MOPU, 1986, 331 pp.

DGPC: Manual de inversiones en puertos, Madrid, MOPU, 1986, 2 vols.

ESTEVE SECALL, R.: «El Frente Portuario Andaluz». Revista de Estudios Regionoles, n. ${ }^{\circ} 13,1984$, pág. 3-29.

GARCIA ALVAREZ, A.: «Criterios metodológicos y normativos para la ordenación integral de las zonas costeras», Revista de Estudios Territoriales, n. ${ }^{\circ}$ 6, 1982, pág. 41-59.

GARCIA ALVAREZ, A.: Ordenación de los espacios litorales. Criterios metodologicos y normativos, Madrid, CEOTMA, 1982, 266 pp.

GONZALES MACIAS, A.: «El puerto Algeciras-La Línea», Jornadas sobre el transporte y comunicaciones en la provincia de Cádiz, Algeciras, 1984, 9 pp.

GONZALEZ MACIAS, A.: «Los puertos andaluces de interés general y su importancia en el contexto de la Comunidad Autónoma», Simposium Territorio, puerto y ciudad, Sevilla, 1986, 12 pp.

GONZALEZ PAZ, J.: «Política de ordenación integrada de los espacios litorales», Revista de Estudios Territoriales, $\mathrm{n}^{\circ}$ 6, 1982, pág. 97-109. 
GROSSO, S.: «Algeciras, primer puerto nacional en pasajeros, vehículos y contenedores», Diario de Cádiz, Suplemento Dominical, 2 de Agosto, 1987, pág. 6-9.

GUERRERO HERNAN, F.: «Los puertos en el transporte de línea regular», Curso Superior de Negocio Marítimo, Madrid, 1986, 19 pp.

HERCE VALLEJO, M.: «La costa, un recurso natural en proceso de deterioro», Revista Ciudad y Territorio, n. ${ }^{\circ}$ 3, 1977, pág. 49-58.

INGENIEROS CONSULTORES S.A. (ICSA): Estudio de las consecuencias socioeconómicas derivadas de la existencia del puerto Algeciras-La Línea, 1982, 363 pp.

INSTITUTO DEL TERRITORIO Y URBANISMO (ITUR): Análisis del litoral español. Diseño de políticas territoriales, Madrid, MOPU, 1987, $243 \mathrm{pp}$.

LEFLER PINO, J.: «Presente y futuro de la Bahía de Algeciras», Revista de Obras Públicas, n. ${ }^{\circ} 288$, 1981, pág. 30-32.

LEFLER PINO, J.: «Estructura portuaria, marítima y fluvial de Andalucía», I Seminario-Coloquio sobre infraestructura y transporte, La Rábida (Huelva), 1982, 12 pp.

LEFLER PINO, J.: «El puerto de Algeciras-La Línea», Jornadas de Estudios Marítimos, Algeciras, $1984,12 \mathrm{pp}$.

LOPEZ CUEVAS, V.J.: «El puerto de Algeciras y sus posibilidades», Simposium Territorio, puerto y ciudad, Sevilla, 1986, 11 pp.

LOZANO MALDONADO, J.M.: El desarrollo del Campo de Gibraltar. Análisis Geográfico de una década decisiva, Málaga, C.A. de Ronda, 1983, 491 pp.

MARTIN ALMENDRO, M.: «Situación del transporte marítimo en la zona surmediterránea y suratlántica», Simposium Territorio, puerto y ciudad, Sevilla, 1986, 4 pp.

MARTIN, J.L., y OTROS: «El puerto de Algeciras, una metodología para el estudio de sus funciones», Revista Geographica, 1983, pág. 27-59.

MCGREGOR NAVIRE: «Emancipation of the rail ferry», Rev. McGregor Navire, п. ${ }^{\circ} 105,1985$, pág. 4-11.

MOORE, G.: «La ampliación portuaria pasa por un entendimiento entre Algeciras, Cádiz y Gibraltar», Diario de Cádiz, 24 de Agosto de 1986, pág. 17.

NUÑEZ, E.: «Los puertos algecireños no están preparados para competir con los europeos», Diario de Cádiz, Suplemento Dominical, 2 de Agosto de 1987, pág. 6-9.

PORT DEVELOPMENT INTERNATIONAL: «Algeciras-especulating», Port Development International, Abril, 1986, pág. 30-31.

POZUETA ECHEVARRI, J.: «El planeamiento ante el espacio portuario, situación y problemática», Ciudad y Territorio, n. ${ }^{\circ} 74,1987$, pág. 57-65.

ROCHER Y VACA, R.: «Asentamiento de grandes complejos industriales», I Curso de análisis, planeamiento y gestión del medio litoral, Madrid, 1974, pág. 93-107.

RODRIGUEZ BARO, R.: «Política portuaria en los puertos de Andalucía», I Jornadas Sindicales de Puertos de Andalucía, Cádiz, 1986, 11 pp.

GARCIA LIZANA, A. y OTROS: «La potenciación de los puertos andaluces y el desarrollo regional. El caso de Algeciras», VIII Reunión de Estudios Regionales, Bilbao, 1982, 9 pp.

ROMANOS GRACIA, C., «Desarrollo integral de la Bahía de Algeciras», I Reunión de Estudios Regionales, Barcelona, pág. 425-436. 
SENDIN GARCIA, M.A.: «Observaciones sobre la ciudad y el puerto de Algeciras», Revista Ería, n. ${ }^{\circ} 6,1983$, pág. 133-157.

SLACK, B.: «Containerization, interport competition and port selection», Rev. Maritime policy and management, n. ${ }^{0} 4,1985$, pág. 293-303.

TRONCOSO SANABRIA, P.: «Estudio sobre el desarrollo del transporte en contenedores de la región suratlántica», Curso monográfico de doctorado en Ciencius del Mar, 1985, 34 pp.

CUADRADO ROURA, J.R.: Estudio económico de la provincia de Cádiz. 